\title{
Other Research
}

\section{Economic consequences of reproductive performance in dairy cattle}

\section{Introduction}

In the dairy industry, good reproductive performance is important. Reproductive performance of individual cows is the result of a physiological interactions, influenced by, for instance, the milk production level of a cow. Reproductive performance may differ from cow to cow. Farmer's decisions, therefore, also may differ from cow to cow. The dairy farmers need practical information for their decisions regarding reproduction. Simulation modeling can be a good tool to support farmers in their decisions. The objective of this work was to create a simulation model to calculate the economic consequences for single cows on farms with average, good and poor reproductive performance and compare them with an imaginary cow having an excellent reproductive performance and calculate the economic benefits of improving individual reproduction parameters.

\section{Materials and Methods}

A Monte-Carlo dynamic stochastic simulation model was created to calculate the economic effects of non-optimal fertility in individual dairy cows. The model is dynamic and uses time steps of one week. The probabilities of events in a certain time step are on the value of input parameters and events in the previous time steps. The default values of the input parameters that were used in the model depended on literature and authors' expertise. The model used enough iterations to reach a steady state, defined as the moment where the SD of output variables changed less than $1.5 \%$ after a new iteration.
The milk production of each cow during each time step was simulated using Wood's function dependingon parity, milkproduction level, week of highest milk production and persistency. The reproductive events, including ovulation, estrous detection, conception and postpartum disorders were predicted using discrete distribution functions with relevant probabilities. Probabilities of these reproductive events were adjusted per time step, based on cow factors such as milk production level, parity and lactation stage. If a cow got pregnant, the milk production was adjusted depending on the gestation stage. Per cow, per year, the milk production, number of calves, number of inseminations and number of culled cows, were summarized and used for further economic analysis.

A partial budget approach, i.e. a calculation of the net effect of changes of a certain situation compared with a basic situation, was used. An imaginary, excellent reproductive performance was used as basic situation and consisted of a $100 \%$ probability for ovulation, oestrus detection and conception and $0 \%$ probability of postpartum reproductive disorders and a voluntary waiting period (VWP) of 6 weeks. Three scenarios of realistic reproductive performances ("good", "average" and "poor") were compared with the "excellent" reproductive performance. The net economic losses (NELi) of these less-than-excellent scenarios were calculated as the change of the sum of additional revenues or revenues forgone (milk production, culling, calves), reduced costs or extra cost (Al, calving management). For the "good" reproductive scenario, values for probabilities of ovulation, oestrus detection, conception, reproductive disorders and voluntary waiting period were $100 \%, 70 \%, 70 \%$, $3 \%$ and 9 weeks, respectively. For the "average" reproductive scenario, values for probabilities of ovulation, oestrus detection, conception, reproductive disorders and voluntary waiting period were $95 \%, 50 \%$, $50 \%, 7 \%$ and 12 weeks, respectively. For the "poor" reproductive scenario, values for probabilities of ovulation, oestrus detection, conception, reproductive disorders and voluntary waiting period were $90 \% 30 \%$, $30 \%, 11 \%$ and 15 weeks, respectively.

The model was validated internally for credibility and validity on the basis of the literature and the authors' expertise. A sensitivity analysis was evaluated by comparing the change of value of a singled parameter in the good and poor scenario with the average scenario.

\section{Results}

Annual economic losses ( $€$ /cow) of "poor", "average" and "good" real reproductive performancescenarios related to a "excellent" reproductive performance scenario (5\%$95 \%$ percentiles in parentheses) are shown in Table 1. The annual economic losses are estimated to be $€ 28, € 88$ and $€ 282$ per cow, respectively, for a "good", "average", and "poor" reproductive performance. Milk production losses, due to fewer calvings per year, were the most important cost factor. However, when the reproductive performance is reduced, the importance of culling increases. In the "poor" reproductive performance scenario, culling was the most important factor.

Table 1: Average and variation (5 and 95 percentiles within brackets) economic losses (€ per cow year) of the "good", "average" and "poor" reproductive scenario as compared with the imaginary excellent reproductive performance.

\begin{tabular}{|c|c|c|c|}
\hline Economic losses & Good & Average & Poor \\
\hline Milk production & $26(3-74)$ & $72(10-207)$ & $128(22-278)$ \\
\hline Calf & $11(3-23)$ & $21(10-38)$ & $30(16-44)$ \\
\hline Culling & $0(0-0)$ & $14(0-0)$ & 187 (0-791) \\
\hline $\mathrm{Al}$ & $3(-3-24)$ & $8(-5-40)$ & $12(-7-60)$ \\
\hline Calving management & $-13(-27-(-4))$ & $-25(-44-(-12))$ & $-35(-51-(-19))$ \\
\hline Total Net & $28(1-88)$ & $88(5-259)$ & $282(19-818)$ \\
\hline
\end{tabular}


There was a large variation in net economic losses due to less-than-excellent reproductive performance (Figure 1). For individual cows, the net economic losses of "good" reproductive performance could vary between $€ 1$ and $€ 88$ per year. For an "average" reproductive performance, the variation was between $€ 5$ and $€ 259$ per year. For a "poor" reproductive performance, the losses for a single cow could vary between $€ 19$ and $€ 818$.

The most important variable influencing the annual net economic losses was the probability of conception, followed by the probability of oestrus, the length of the VWP, the probability of ovulation after the first ovulation and the incidence of postpartum disorders.

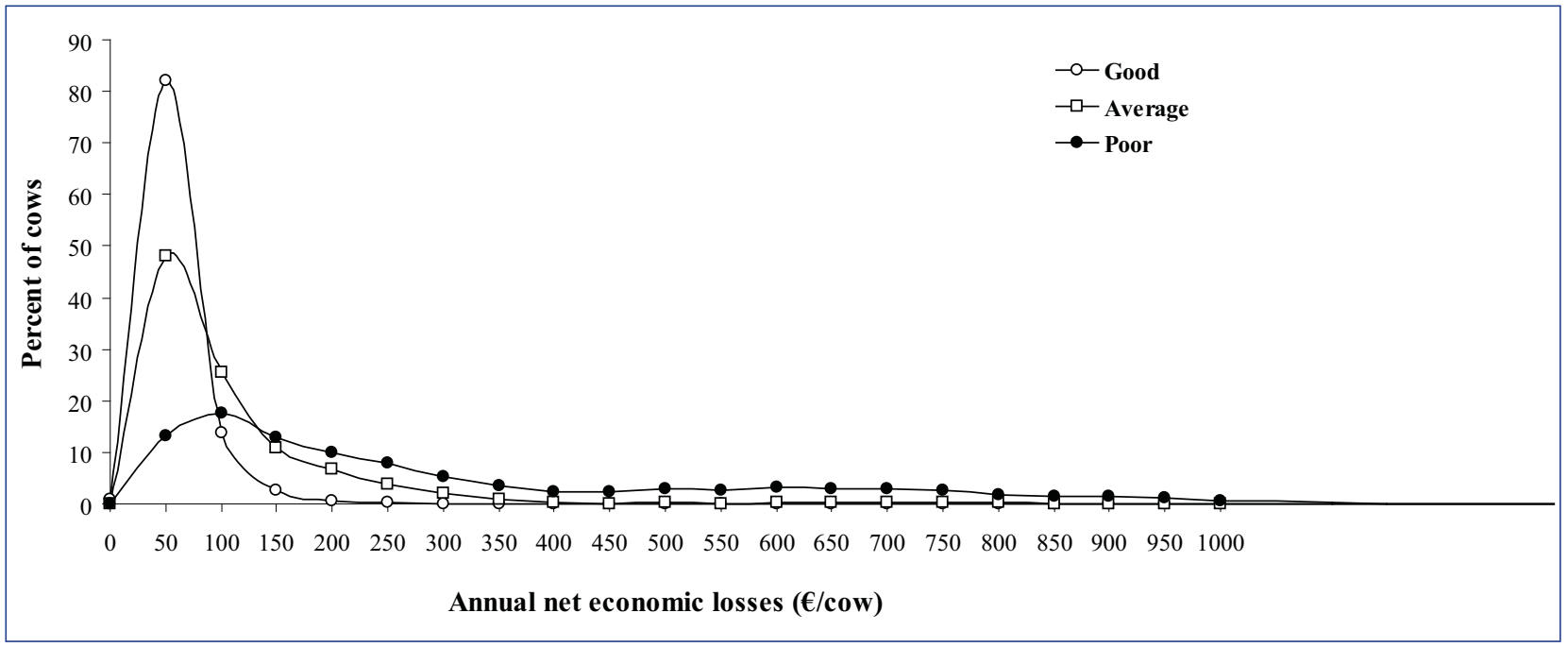

Figure 1. Percentage of cows at different average of annual net economic losses (€/cow) of "poor", "average" and "good" reproductive performance scenarios related to the "excellent" reproductive performance scenario.

\section{Conclusion}

Non optimal fertility, defined as a "good", "average", or "poor" reproductive performance, resulted in mean net losses compared to an imaginary "excellent" reproductive performance of $€ 28, € 88$ and $€ 282$ per cow per year, respectively. These losses could vary largely between cows and are mainly caused by decreased milk production and increased culling rate, especially in the situation of poor fertility.
Chaidate Inchaisri, Ruurd Jorritsma, Peter L.A.M. Vos, Bert van der Weijden and Henk Department of Farm Animal Health, Faculty of Veterinary Medicine, Utrecht University, The Netherlands

e-mail: c.inchaisri@uu.nl

\section{Prevalence of Streptococcus agalactiae in Swedish AMS}

In Sweden SCC is significantly $15 \%$ higher on farms with automatic milking (AMS) compared to the geometric mean in controlled herds. Since sporadic reports from AMS also have indicated an increase of mastitis caused by Str. agalactiae (SRA) a screening of herd prevalence of the bacteria was performed during the first 6 months of 2010.

\section{Method}

Bulk tank samples were taken out manually in tubes with bronopol by veterinarians or technicians. The samples were sent by mail to National Veterinary Institute in Uppsala and frozen upon arrival at the laboratory. The frozen samples were packed and sent chilled to the Finnzyme Laboratory in Finland and tested with PathoProof Mastitis PCR Assay for content of mastitis bacteria. A total number of 465 samples were collected, corresponding to $84 \%$ of the AMS herds in Sweden.

\section{Results}

SRA and Staphylococcus aureus were detected in 23 and 221 of the herds respectively. This means that the herd prevalence for SRA in this investigation was $5 \%$ and that Staph. aureus was at hand in every other Swedish AMS. (See Figure 1).

\section{Discussion}

The PCR test chosen detects 11 different mastitis bacteria and the B-lactamase gene. The clinical relevance is assumed to be higher for contagious bacteria with their reservoir within the udder. For environmental microbes contamination can be an important additional source. For this reason no results other than for SRA and Staph. aureus are presented in this report.

The prevalence level for SRA is quite similar to the result obtained in Denmark (6\%) where all DHI herds have been tested for SRA during the last year.

Importantexperiencesin Swedisheradication of SRA is that the transition period is vital and often with inadequate biosecurity. Another remark is that screening, culling and treatment are of no use without a functional segregation plan. 